
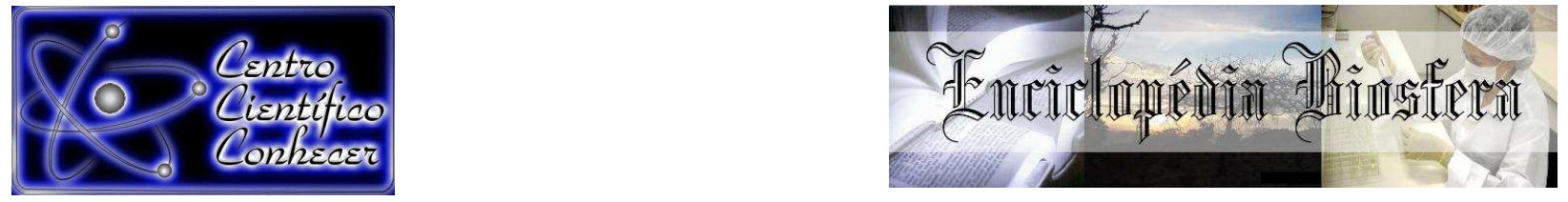

\title{
CASOS DE HANSENÍASE ENTRE OS ANOS DE 2010 E 2014 NO MUNICÍPIO DE ALTA FLORESTA, MATO GROSSO, BRASIL
}

Izailde Ribeiro da Silva Novakoski ${ }^{1}$, Linda Mara Alvino dos santos Ximenes ${ }^{2}$, José Martins Fernandes ${ }^{3}$

1 Graduanda em Licenciatura Plena em Ciências Biológicas, Campus Universitário de Alta Floresta, Universidade do Estado de Mato Grosso (UNEMAT)

2 Graduada em Licenciatura Plena em Ciências Biológicas, Campus Universitário de Alta Floresta, UNEMAT

3 Professor da Faculdade de Ciências Biológicas e Agrárias, Campus Universitário de Alta Floresta, UNEMAT (fernanbio@bol.com.br)

Recebido em: 08/04/2016 - Aprovado em: 30/05/2016 - Publicado em: 20/06/2016 DOI: 10.18677/Enciclopedia_Biosfera_2016_130

A Hanseníase é uma doença contagiosa causada pelo Mycobacterium leprae, que afeta a pele, sistema nervoso periférico e também outros órgãos e sistemas do corpo humano. O trabalho teve como objetivo analisar os casos de hanseníase no município de Alta Floresta, Mato Grosso, no período entre 2010 e 2014. O presente estudo promoveu o levantamento clinico-epidemiológico dos casos de hanseníase notificados pela Vigilância Epidemiológica. Os casos foram analisados quanto: ao sexo dos pacientes, área de maior ocorrência, idade dos pacientes, faixa etária mais atingida, escolaridade, número de casos por unidade de saúde e classificação das formas em Multibacilar e Paucibacilar. Dos 862 casos de hanseníase notificados, $54,18 \%$ foram em pessoas do sexo feminino, a maioria reside na área urbana $(87 \%)$, com maior faixa etária entre 20 e 49 anos (55,46\%). A escolaridade dos pacientes é baixa, principalmente com o Ensino Fundamental incompleto (54,53\%). Foram notificados casos em 12 PSFs (Programa de Saúde da Família) e 2 postos de saúde, sendo o PSF II no bairro Cidade Bela com maior incidência (143 casos, $16,59 \%$ ). Destacou-se a forma Paucibacilar em 56,03\%, com até cinco lesões. Os dados adquiridos apontam um número maior de casos de Hanseníase em mulheres o que diferencia a pesquisa em relação a outros trabalhos que observaram índices mais elevados em homens. Também sugere que o maior número de casos no município de Alta Floresta está relacionado às condições socioeconômicas.

PALAVRAS-CHAVE: Bairro Cidade Bela, Lepra, Mycobacterium leprae.

\section{BETWEEN LEPROSY CASES THE YEARS 2010 AND 2014 IN THE MUNICIPALITY OF ALTA FLORESTA, MATO GROSSO, BRAZIL}

\begin{abstract}
Leprosy is a contagious disease caused by Mycobacterium leprae, which affects the skin, peripheral nervous system and also other organs and body systems. The study aimed to analyze the leprosy cases in the municipality of Alta Floresta, Mato ENCICLOPÉDIA BIOSFERA, Centro Científico Conhecer - Goiânia, v.13 n.23; p.216 2016
\end{abstract}


Grosso, in the period between 2010 and 2014. This study promoted the clinicoepidemiological study of leprosy cases notified by the Epidemiological Surveillance. The cases were analyzed: gender of patients, higher incidence area, age of patients, most affected age group, education level, number of cases per health unit and classification of forms in multibacillary and paucibacillary. Of the 862 cases of leprosy reported, $54.18 \%$ were female people, most reside in urban areas $(87 \%)$, with most aged between 20 and 49 years $(55.46 \%)$. The education of patients is low, especially with incomplete primary education (54.53\%). cases were reported in 12 PSF (Family Health Program) and 2 health centers, with PSF II in the City Beautiful neighborhood with the highest incidence (143 cases, $16.59 \%$ ). He stood out in paucibacillary form $56.03 \%$, with up to five lesions. The acquired data indicate a greater number of leprosy cases in women what differentiates research in relation to other studies that found higher rates in men. It also suggests that the largest number of cases in the municipality of Alta Floresta is related to socioeconomic conditions.

KEYWORDS: Neighborhood Cidade Bela, Leprosy, Mycobacterium leprae.

\section{INTRODUÇÃO}

A hanseníase é causada pela espécie de bactéria Mycobacterium leprae, também conhecida como bacilo de Hansen, que é um parasita intracelular obrigatório, com afinidade por células cutâneas; é uma doença infectocontagiosa, de evolução lenta, que se manifesta principalmente através de sinais e sintomas dermatoneurológicos, que são lesões na pele e nos nervos periféricos, principalmente nos olhos, mãos e pés (BRASIL, 2002a; PEREIRA et al., 2012).

Ainda no século XXI, a detecção de casos novos de hanseníase permanece elevada no mundo, com cerca de 250 mil casos registrados a cada ano (MONTEIRO et al., 2013). No Brasil, a hanseníase tem sido incluída, pelo próprio país, entre outras doenças referidas, no sexto objetivo de desenvolvimento do milênio devido ao país ser considerado o segundo do mundo em número de casos, perdendo apenas para a Índia (BRASIL, 2010; HACKER et al., 2012). Usualmente, atinge populações menos favorecidas (SILVA et al., 2010) com desigualdades sociais e com pouca atenção do poder público. O controle da doença está baseado no diagnóstico precoce e no tratamento constante, visando eliminar o mais rápido possível as fontes de transmissão da doença (BRASIL, 2010).

A hanseníase é uma doença concentrada principalmente nas regiões Norte e Centro-Oeste do Brasil (BRASIL, 2010; FERREIRA et al., 2012; GARCIA et al., 2013). O quadro epidemiológico de Mato Grosso revela elevada prevalência da doença, alcançando valores acima de 24 casos por 10.000 habitantes (IGNOTTI et al., 2004).

A contaminação por hanseníase é de pessoa para pessoa e ocorre através das vias respiratórias, secreções nasais ou saliva (CID et al., 2012). A bactéria tem alta efetividade e baixa patogenicidade, isto é, infectam várias pessoas, no entanto só poucas adoecem e manifesta os sintomas (BRASIL, 2002a). O tempo de multiplicação do bacilo é lento, podendo durar, em média, de 11 a 16 dias (BRASIL, 2002). Tem um período médio de incubação que vai de dois a sete anos, embora possa também apresentar períodos curtos de sete meses e longos de até 10 anos (SANTOS et al., 2005). 
O homem e alguns animais silvestres como tatu, macaco mangabei e chipanzé são considerados as únicas fontes de infecção da hanseníase, e no caso dos humanos o contágio acontece através de uma pessoa doente, portadora do bacilo de Hansen, não tratada, que o elimina para o meio exterior, contagiando pessoas susceptíveis (BRASIL, 2002b). O acesso à poliquimioterapia mudou drasticamente a perspectiva de vida das pessoas atingidas pela hanseníase e significou liberação de recursos para investimento em outros problemas de saúde (BRASIL, 2010). O reconhecimento precoce e o tratamento oportuno são elementos chave para cessar a transmissão, prevenindo incapacidades no portador (LASTORIA \& ABREU, 2012).

LASTORIA \& ABREU (2012) citam que fatores preditivos à ocorrência de recidiva relacionam-se com condições de moradia, hábitos de vida, organização dos serviços de saúde, formas clínicas e esquemas terapêuticos, como medidas de prevenção da recidiva, cabe aos serviços de saúde oferecer orientações adequadas aos pacientes, bem como, garantir a regularidade do tratamento.

Segundo ARAÚJO (2003), a predileção pela pele e nervos periféricos confere características peculiares a esta moléstia, tornando o diagnóstico simples na maioria dos casos. Sabe-se que o bacilo aloja-se nos nervos periféricos, provocando manchas e nódulos, principalmente no rosto, ulcerações nos pés e nas mãos, perda de pêlos, inclusive os da sobrancelha e dos cílios, insensibilidade das extremidades entre outros sintomas (CUNHA, 1997).

A grande reorganização da assistência aos pacientes com hanseníase iniciou-se na década de 80 pela Secretaria de Ações Básicas de Saúde, que recebeu um importante impulso da Secretaria Nacional de Programas Especiais de Saúde passando pela Secretaria de Assistência à Saúde e pela Fundação Nacional de Saúde, onde um enorme esforço vem sendo feito desde então, de forma continuada, envolvendo um verdadeiro arsenal de profissionais de saúde em todo o país (BRASIL, 2002a). O diagnóstico de casos de hanseníase na atenção básica de saúde é essencialmente clínico por meio do exame dermatoneurológico para identificar lesões ou áreas de pele com alteração de sensibilidade e/ou comprometimento de nervos periféricos (BRASIL, 2007).

CUNHA (1997) cita em sua pesquisa que a hanseníase é considerada ainda de baixa letalidade, isto é, dificilmente leva à morte, por outro lado, é uma doença altamente incapacitante pelas lesões que provoca nos nervos, afetando a motricidade, deixando sequelas nas mãos, pés e olhos, tornando impossível a locomoção e o trabalho manual, podendo levar ainda à cegueira. Por causa disso, o doente é afetado também como ser social, uma vez que suas relações sociais também são prejudicadas. As pessoas com quem convive, tanto na família quanto no trabalho, tendem a isolá-lo, tornando-o marginalizado e inoperante na sociedade, fenômeno que ocorre de forma semelhante com outras doenças e deficiências. Segundo SAVASSI (2010), a hanseníase é uma doença de notificação compulsória que está inclusa no rol das doenças negligenciadas.

É fácil de ser diagnosticada, tratada e curada, no entanto, quando diagnosticada e tratada tardiamente pode trazer graves consequências para os portadores e seus familiares pelas lesões que os incapacitam fisicamente (BRASIL, 2002b). De acordo com SOUZA (1997) a hanseníase é classificada em Paucibacilares (PB), casos com até cinco lesões de pele e Multibacilares (MB), casos com mais de cinco lesões de pele. Segundo os mesmos autores, o ENCICLOPÉDIA BIOSFERA, Centro Científico Conhecer - Goiânia, v.13 n.23; p.218 2016 
diagnóstico da doença e a classificação operacional do paciente em Paucibacilar ou em Multibacilar é importante para que possa ser selecionado o esquema de tratamento quimioterápico adequado. Segundo RAMOS et al. (2013) entre os óbitos devido a hanseníase, apenas $15 \%$ são de casos classificados operacionalmente como paucibacilares.

O trabalho teve como objetivo analisar os casos de hanseníase no município de Alta Floresta, Mato Grosso, no período entre 2010 e 2014. Além de relacionar o número de casos com informações socioeconômicas dos portadores da doença.

\section{MATERIAL E MÉTODOS}

\section{Área de Estudo}

A área de pesquisa compreendeu o município de Alta Floresta, Mato Grosso, que fica aproximadamente a $830 \mathrm{~km}$ da capital do estado, Cuiabá, e encontra-se no

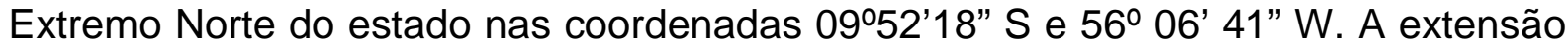
territorial é de aproximadamente $8.976,204 \mathrm{Km}^{2}$ e limita-se com os municípios de Tabaporã, Novo Mundo, Juara, Nova Canaã do Norte, Nova Monte Verde, Carlinda, Paranaíta e o Estado do Pará, com uma população estimada para o ano de 2014 em 49.877 habitantes (SEPLAN, 2014; IBGE, 2015).

Esse município, assim como outros da região, teve colonização recente, iniciada na década de 1970, dependente de atividades baseadas na extração madeireira, agricultura, garimpo e, mais recentemente tornou-se polo da atividade pecuária da região Norte do Mato Grosso, contando com 828.350 cabeças de gado (SEPLAN, 2014; IBGE, 2015). O município também se destaca como polo universitário, recebendo estudantes principalmente de Mato Grosso e estados circunvizinhos.

A vegetação natural predominante no município de Alta Floresta classifica-se em Floresta Ombrófila Densa, Floresta Ombrófila Aberta e Floresta Estacional Semidecidual (ZAPPI et al., 2011; ALMEIDA et al., 2014; LOPES-SOARES et al., 2014; FERNANDES et al., 2015). O clima é quente e úmido, com nítida estação seca; apresenta temperaturas entre $20^{\circ} \mathrm{C}$ a $38^{\circ} \mathrm{C}$, tendo em média $26^{\circ} \mathrm{C}$, podendo atingir nos dias mais quentes temperaturas superiores a $40^{\circ} \mathrm{C}$ (SEPLAN, 2014).

\section{Procedimentos Metodológicos}

Os dados da pesquisa foram fornecidos entre dezembro de 2014 e maio de 2015, pela coordenação da Vigilância Epidemiológica (VIGEP) da Secretaria Municipal de Saúde de Alta Floresta, Mato Grosso, referente aos casos de Hanseníase entre os anos de 2010 e 2014 no município de Alta Floresta, Mato Grosso.

A VIGEP está situada entre as Avenidas Ariosto da Riva Neto e Ludovico da Riva Neto, canteiro central em Alta Floresta, Mato Grosso, responsável pelo controle e envio de relatórios das notificações de casos de Hanseníase à Secretaria Estadual de Saúde.

A pesquisa foi fundamentada na utilização de dados escolhidos em 862 fichas de notificação considerando: sexo dos pacientes em tratamento (masculino ou feminino); área de maior ocorrência (zona rural, urbana ou Peri urbana=perímetro urbano); idade dos pacientes, e qual a faixa etária mais atingida; escolaridade; 
número de casos por unidade de saúde; classificação das formas em Multibacilar e Paucibacilar. Os dados foram organizados em tabelas e figuras e discutidos com o auxílio de informações disponíveis na leitura da área.

\section{RESULTADOS E DISCUSSÃO}

Foram notificados entre 2010 e 2014, 862 casos de hanseníase no município de Alta Floresta, Mato Grosso. A maior incidência ocorreu em mulheres somando um total de 467 pacientes (54,18\%), enquanto que $395(45,82 \%)$ casos foram notificados em homens. Os dados apontam para um número maior de casos em mulheres, que diferencia a pesquisa em relação a outros trabalhos anteriores que observaram índices mais elevados da doença entre os homens (BRASIL, 2002a; MONTEIRO et al., 2013; RAMOS et al., 2013).

Entre os casos notificados a maior parte das pessoas reside na área urbana (87\%), conforme Figura 1. A maior incidência de casos de hanseníase na zona urbana também é corroborada em outras pesquisas (EVANGELISTA, 2004; DIAS et al., 2005). No município de Alta Floresta, cerca de $86 \%$ da população reside na zona urbana (IBGE, 2015), influenciando diretamente no número de casos notificados.

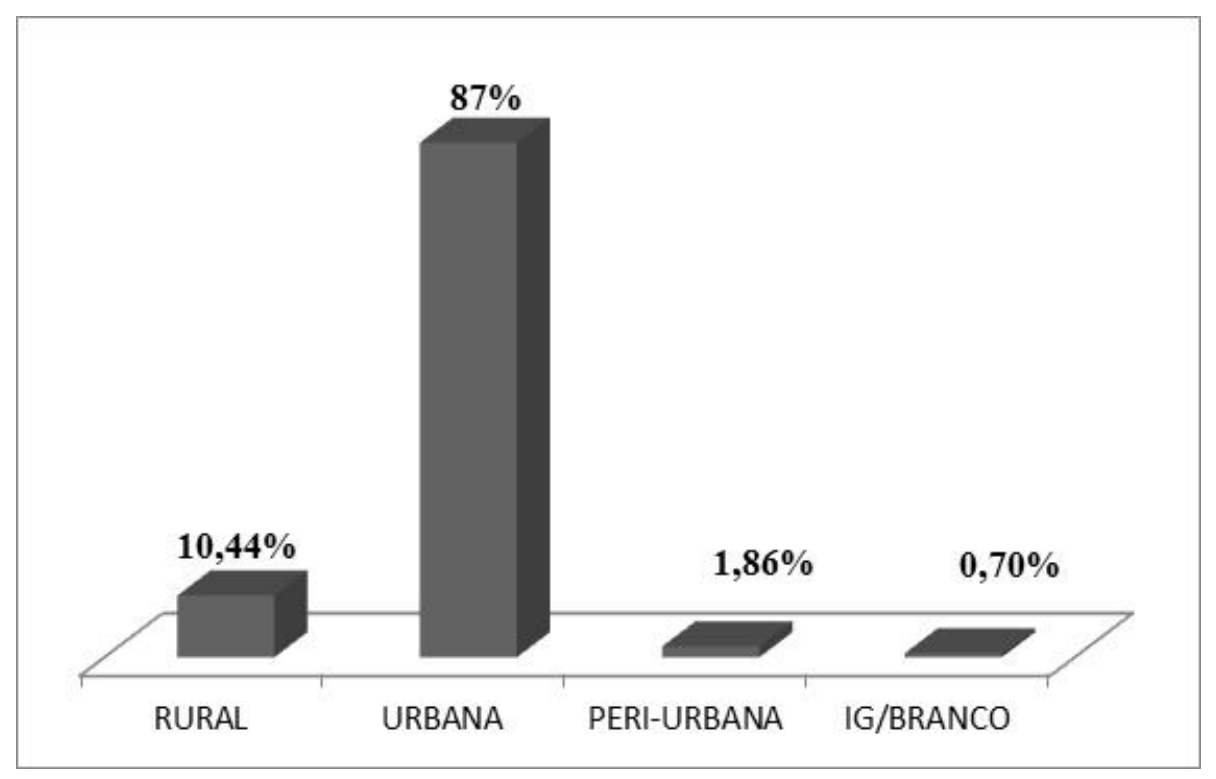

FIGURA 1. Local de residência dos portadores de Hanseníase entre 2010 e 2014 no Município de Alta Floresta, Mato Grosso. Peri-urbana=perímetro urbano, IG/branco=dados em branco.

Na Figura 2, observa-se que o maior número de casos ocorreu entre 20 e 49 anos (55,46\%), seguido entre 50 e 79 anos (31,20\%). Em pesquisa realizada no Estado de Santa Catarina, MELÃO et al. (2011) observaram o maior acometimento entre a faixa etária de 30 a 39 anos. Segundo dados do Ministério da Saúde, a hanseníase pode atingir pessoas de todas as idades, de ambos os sexos, no entanto, raramente ocorre em crianças, enquanto a maior incidência da doença ocorre em adultos do sexo masculino do que no sexo feminino, na maioria das regiões do mundo (BRASIL, 2002a). 


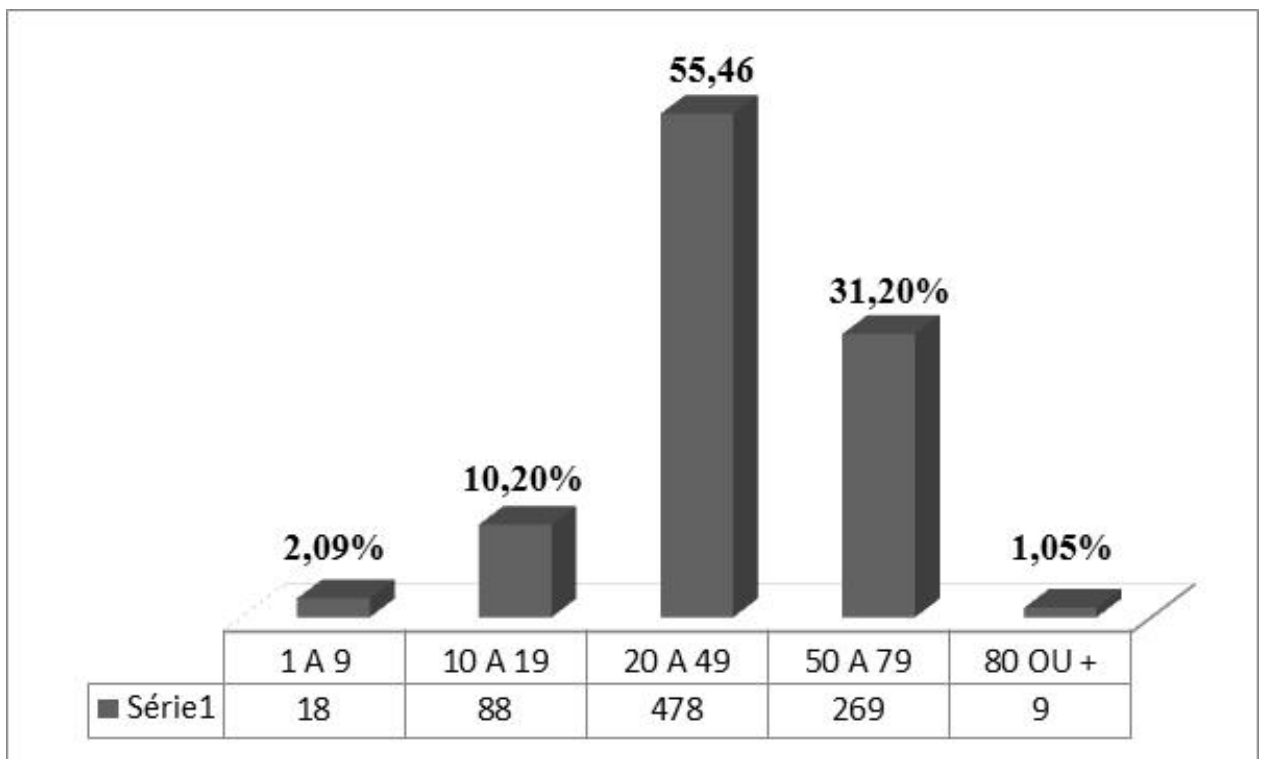

FIGURA 2. Número de casos e faixa etária dos pacientes notificados com hanseníase entre os anos de 2010 e 2014 no município de Alta Floresta, Mato Grosso.

A Figura 3 mostra que $54,53 \%$ das pessoas com notificações de hanseníase possuem apenas o Ensino Fundamental Completo (EFC). Segundo RAMOS et al. (2013), em relação aos aspectos sociais dos indivíduos com hanseníase, cerca de $60 \%$ possuem baixo nível de escolaridade. Dados que também estão de acordo com o encontrado no município de Alta Floresta.

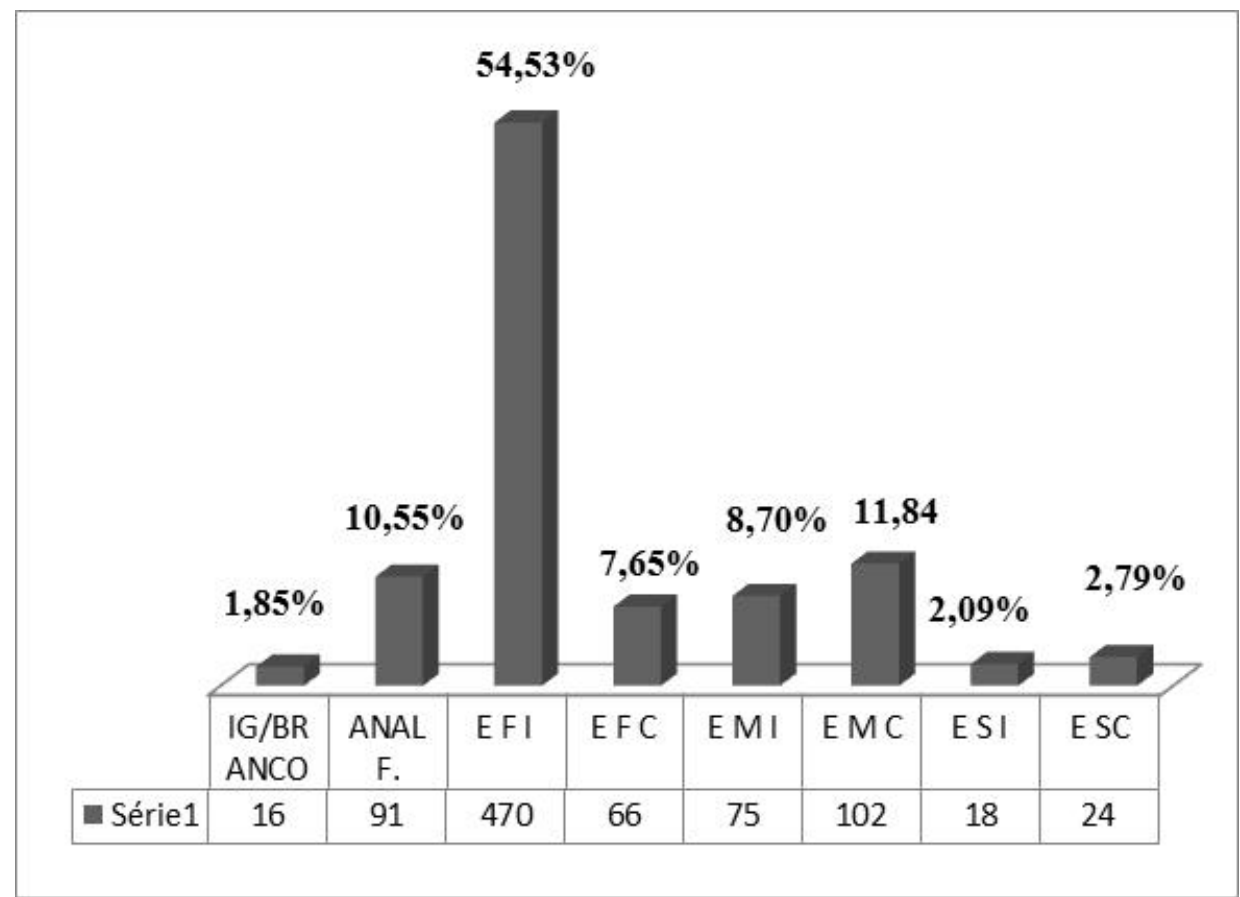

FIGURA 3. Escolaridade dos pacientes com hanseníase no município de Alta Floresta, Mato Grosso, entre 2010 e 2014. 
A Tabela 1 demonstra o total de casos de hanseníase ocorridos entre 2010 e 2014 em PSFs (Programa de Saúde da Família) e Postos de Saúde do município de Alta Floresta. No PSF II localizado no bairro Cidade Bela foi constatado o maior número com 143 casos (16,58\%), PSF I no bairro Vila Nova com 120 casos $(13,92 \%)$, seguido pelo Posto Santa Barbara situado no Setor G, com 114 casos $(13,22 \%)$. No PSF VII, que atende moradores da zona rural, foram notificados apenas 30 casos $(3,49 \%)$. Os bairros Cidade Bela e Vila Nova localizam-se no entorno do centro urbano do município de Alta Floresta, estando entre os primeiros bairros do município.

TABELA 1. Número de casos de hanseníase notificados entre 2010 e 2014 nos postos de saúde no município de Alta Floresta, Mato Grosso.

\begin{tabular}{l|cll}
\hline \multicolumn{1}{c}{ POSTO DE SAÚDE } & NNo DE & \multicolumn{1}{c}{ BAIRRO } & CÓDIGO \\
& CASOS & & \\
\hline Julia Maria da Silva & 143 & Cidade Bela & PSF II \\
Vila Nova & 120 & Vila Nova & PSF I \\
Posto Santa Barbara & 114 & Setor G & POSTO \\
Posto Ana Neri & 86 & Setor R I & POSTO \\
Santa Rita de Cássia & 65 & Bom Pastor & PSF IV \\
Jardim Panorama & 58 & Jardim Panorama & PSF III \\
Cidade Alta II & 51 & Setor Norte três & PSF XII \\
Boa Esperança & 43 & Boa Esperança & PSF VIII \\
Bom Jesus & 37 & Bom Jesus & PSF IX \\
Enfermeiro Gileno F. Teófilo & 37 & São Jose Operário & PSF X \\
Valfredo J. Santana & 35 & Setor Universitário & PSF XI \\
Zona Rural & 30 & Zona rural & PSF VII \\
Professor Dione L. B. dos Santos & 24 & Boa Nova & PSF VI \\
Cidade Alta & 19 & Cidade Alta & PSF V \\
\hline
\end{tabular}

Segundo ABRAÇADO et al. (2015) adultos de baixa renda e baixa escolaridade são achados comuns nos trabalhos epidemiológicos de hanseníase. Para BRITO et al. (2014), entre as premissas sociais associadas à distribuição geográfica da doença, reafirmam-se a pobreza, a desnutrição ou algumas carências nutricionais, além de condições higiênicas desfavoráveis e movimentos migratórios, Informações estas que relacionam as maiores incidências de hanseníase com as baixas condições socioeconômicas e escolaridades.

A Figura 4 demonstra que entre os casos notificados, 483 pacientes $(56,03 \%)$ apresentaram duas a cinco lesões, sendo considerados como Paucibacilar (PB), com cinco ou mais lesões foram 237 pessoas $(27,50 \%)$, considerados Multibacilar (MB). Os dados demonstram que os portadores da doença procuram assistência médica quando as primeiras lesões aparecem no corpo, mesmo assim, o número de portadores com mais de cinco lesões ainda é considerado muito elevado. Porém, os dados de Alta Floresta contrapõem a afirmação de SILVA et al. (2010), onde citam que a frequência de pacientes com a forma Multibacilar da doença é maior, com mais de cinco lesões. 


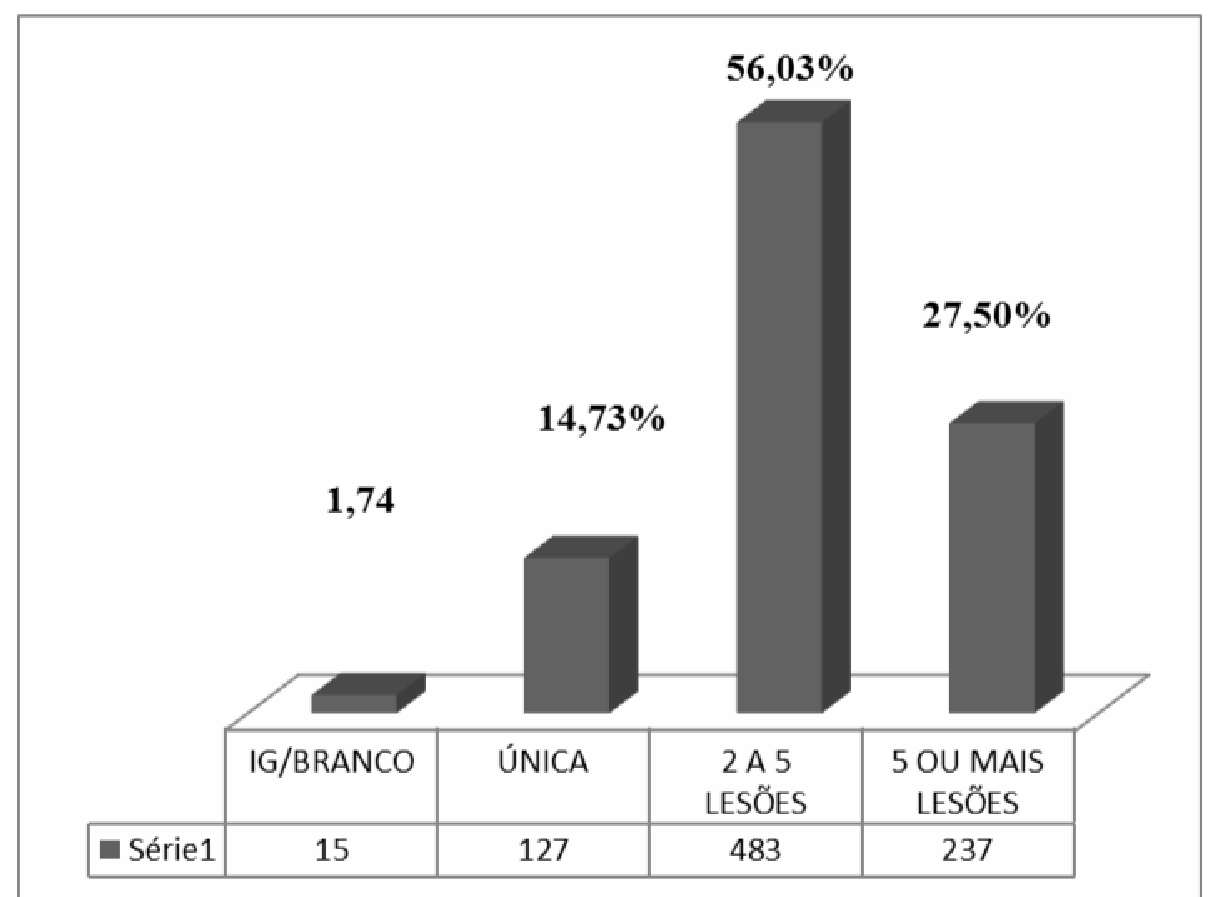

FIGURA 4. Números de casos de hanseníase Multibacilar (MB) e Paucibacilar (PB) no município de Alta Floresta, Mato Grosso, no período de 2010 e 2014.

Segundo NASCIMENTO et al. (2011) o diagnóstico da hanseníase, em grande parte do Brasil, ainda é tardio, cerca de um ano e meio a dois anos após o aparecimento dos sintomas. Segundo os mesmos autores, as campanhas realizadas pelos órgãos da saúde têm como objetivo, não só a orientação de identificação dos sinais e sintomas da doença, mas, sobretudo, um adequado enfrentamento da mesma mediante condutas eficazes para manutenção da saúde e, consequentemente, para a qualidade de vida.

Desta forma, ações de prevenção de incapacidades ou ações de autocuidado em hanseníase têm por objetivo evitar as possíveis deformidades e incapacidades, buscando interromper a propagação das perdas funcionais e sociais, como também o estigma atrelado à doença (SANTOS et al., 2012; DUARTE et al., 2014).

\section{CONCLUSÕES}

A partir deste trabalho, fica evidente que esta doença é um grave problema de saúde pública no município de Alta Floresta, Mato Grosso, principalmente em bairros localizados no entorno do centro urbano, destacando-se o sexo feminino. No entanto, ficou claro que grande maioria dos portadores da doença busca tratamento quando as primeiras lesões aparecem no corpo. Mesmo com os avanços das políticas públicas no combate da doença pelo Ministério da Saúde e dos órgãos competentes de Alta Floresta, ainda é necessário maior divulgação perante a sociedade sobre esta doença, bem como, formas de tratamento, que é simples e gratuito. 


\section{AGRADECIMENTOS}

Aos funcionários da Vigilância Epidemiológica da Secretaria Municipal de Saúde do município de Alta Floresta, Mato Grosso, pelo fornecimento dos dados.

\section{REFERÊNCIAS}

ABRAÇADO, M.F.S.; CUNHA, M. H. C. M.; XAVIER, M. B. Adesão ao tratamento de hanseníase em pacientes com episódios reacionais hansênicos em uma unidade de referência. Revista Pan-Amazônica de Saúde v. 6, n.2, p. 23-28, 2015.

Doi: 10.5123/S2176-62232015000200003.

ALMEIDA, E. A.; SOARES-LOPES, C. R. A.; RODRIGUES, L.; SIMÃO, S. S.; FERNANDES, J. M. Estrutura fitossociológica de floresta estacional decidual submontana e floresta ombrófila aberta submontana em Alta Floresta, Mato Grosso. Enciclopédia Biosfera, v. 10, n.19, p. 1058-1078, 2014.

ARAÚJO, M. G. Hanseníase no Brasil. Revista da Sociedade Brasileira de Medicina Tropical, v. 36, n. 3, p. 373-382, 2003.

BRASIL. Guia para o Controle da hanseníase. Departamento de Atenção Básica. Brasília: Ministério da Saúde, Série A. normas e Manuais Técnicos, n. 11, p. 12-13, 2002a.

BRASIL. Fundação Nacional de Saúde. Guia de vigilância epidemiológica Fundação Nacional de Saúde. 5. ed. Brasília, Ministério da Saúde, p. 12, 2002b.

BRASIL. Ministério da Saúde. Secretaria de Atenção à Saúde. Departamento de Atenção Básica. Vigilância em Saúde: Dengue, Esquistossomose, Hanseníase, Malária, Tracoma e Tuberculose. Brasília: Ministério da Saúde, p. 71, 2007.

BRASIL. Programa Nacional de Hanseníase Situação epidemiológica da Hanseníase no Brasil. Brasília: Ministério da Saúde, p. 11-12, 2010.

BRITO, K. K. G.; ARAÚJO, D. A. L.; UCHÔA, R. E. M. N.; FERREIRA, J. D. L.; SOARES, M. J. G. O.; LIMA, J. O. Epidemiologia da hanseníase em um estado do Nordeste Brasileiro. Revista de Enfermagem da UFPE online, v. 8, n.8, p. 2686 2693, 2014. DOI: 10.5205/reuol.6081-52328-1-SM.0808201415.

CID, R. D. S; LIMA, G. G; SOUZA, A. R; MOURA, A. D. A. Percepção de usuários sobre 0 preconceito da hanseníase. Revista da Rede de Enfermagem do Nordeste, v. 13, n. 5, p. 1004-1012, 2012.

CUNHA, A. Z. S. C. Hanseníase: a história de um problema de saúde pública.

Dissertação de Mestrado. Universidade de Santa Cruz do Sul, 1997. 
DIAS, M, C, F,S; DIAS, G, H; NOBRE, M, L. Distribuição espacial da hanseníase no município de Mossoró/RN, utilizando o Sistema de Informação Geográfica - SIG. Anais Brasileiros de Dermatologia, v. 80, puplemento 3, p. 289-294, 2005.

DUARTE, L. M. C. P. S.; SIMPSON, C. A.; SILVA, T. M. S.; MOURA, I. B. L.; ISOLDI, D. M. R. Ações de autocuidado de pessoas com hanseníase. Revista de Enfermagem da UFPE online, v. 8, n.8, p. 2816-2822, 2014.

DOI: 10.5205/reuol.6081-52328-1-SM.0808201432.

EVANGELISTA, C. M. N. Fatores sócio-econômicos e ambientais relacionados à hanseníase no estado do Ceará. 89 f. Dissertação de mestrado, Universidade Federal do Ceará, Fortaleza, 2004.

FERNANDES, J. M.; LOPES-SOARES, C. R. A.; RIBEIRO, R. S. \& SILVA, D. R. Leguminosae no acervo do Herbário da Amazônia Meridional, Alta Floresta, Mato Grosso. Enciclopédia Biosfera, v. 11, n.21, p. 2272-2293, 2015.

FERREIRA, S. M. B.; IGNOTTI, E.; GAMBA, M. A. Clinical and laboratory characteristics in the retreatment of leprosy relapse. Revista Brasileira de Epidemiologia v. 15, n.3, p. 573-581, 2012.

GARCIA, D.R.; IGNOTTI, E.; CORTELA, D.C.B.; XAVIER, D.R.; BARELLI, C.S.G.A.P. Análise espacial dos casos de hanseníase, com enfoque à área de risco, em uma unidade básica de saúde no município de Cáceres (MT). Caderno de Saúde Coletiva, v. 21, n.2, p. 168-172, 2013.

HACKER, M. A. V. B.; SAlES, A. M.; ALBUQUERQUE, E. C. A.; RANGEL, E.; NERY, J. A. C.; DUPPRE, N. C.; SARNO, E. N. Pacientes em centro de referência para Hanseníase: Rio de Janeiro e Duque de Caxias, 1986-2008. Ciência \& Saúde Coletiva, v. 17, n.9, p. 2533-2541, 2012.

IBGE, Instituto Brasileiro de Geografia e Estatísticas, 2010. Disponível em< http://www.cidades.ibge.gov.br/xtras/temas.php?lang=\&codmun=510025\&idte $\mathrm{ma}=135 \&$ search=mato-grosso|alta-floresta|pecuaria-2013 > acessado em $27 / 05 / 2015$.

IGNOTTI, E.; RODRIGUES, A. M.; ANDRADE, V. L. G.; VALENTE, J. G. Aplicação de métodos de estimativa da prevalência de hanseníase no Estado de Mato Grosso. Revista Brasileira de Epidemiologia, v. 7, n.2, p. 155-166, 2004.

LASTÓRIAI, J. C; ABREU, M. A. M. M. Hanseníase: diagnóstico e tratamento. Botucatu: Editora do Ministério da Saúde, p. 173-179, 2012.

LOPES-SOARES, C. R. A.; RIBEIRO, R. S.; RODRIGUES, L.; CABRAL, F. F.; SILVA, D. R. Check list de Angiospermas da Região de Influência da UHE Sinop, Médio Teles Pires, Mato Grosso. Enciclopédia Biosfera, v. 10, n.19, p. 2036-2048, 2014. 
MELÃO, S.; BLANCO, L. F. O.; MOUNZER, N.; VERONEZI, C. C. D.; SIMÕES, P. W. T. A. Perfil epidemiológico dos pacientes com hanseníase no extremo sul de Santa Catarina, no período de 2001 a 2007. Revista da Sociedade Brasileira de Medicina Tropical, v. 44, n.1, 79-84, 2011.

MONTEIRO, L. D.; ALENCAR, C. H. M.; BARBOSA, J. C.; BRAGA, K. P.; CASTRO, M. D.; HEUKELBACH, J. Incapacidades físicas em pessoas acometidas pela hanseníase no período pós-alta da poliquimioterapia em um município no Norte do Brasil. Caderno de Saúde Pública, v. 29, n.5, p. 909-920, 2013.

NASCIMENTO, G. R. C.; BARRÊTO, A. J. R.; BRANDÃO, G. C. G.; TAVARES, C. $M$. Ações do enfermeiro no controle da hanseníase. Revista de Enfermagem da UFPE online, v. 13, n.4, p. 743-750, 2011.

PEREIRA, D. L.; BRITO, L. M.; NASCIMENTO, A. H.; RIBEIRO, E. L.; LEMOS, K. R. M.; ALVES, J. N.; BRANDÃO, L. C. G. Estudo da prevalência das formas clínicas da hanseníase na cidade de Anápolis- GO. Ensaios e Ciência: Ciências Biológicas, Agrárias e da Saúde, v. 16, n. 1, p. 55-67, 2012.

RAMOS, A.R.S.; FERREIRA, S.M.B.; IGNOTTI, E. Óbitos por hanseníase como causa básica em residentes no Estado de Mato Grosso, Brasil, no período de 2000 a 2007. Epidemiologia e Serviços de Saúde, v. 22, n.2, p. 273-284, 2013. Doi: 10.5123/S1679-49742013000200009.

SANTOS, A. K.; RIBEIRO, A. P. G.; MONTEIRO, S. Hanseníase e práticas da comunicação: estudo de recepção de materiais educativos em um serviço de saúde no Rio de Janeiro. Comunicação, Saúde e Educação, v.16, n.40, p. 205-218, 2012.

SANTOS, A. P. T.; ALMEIDA, G. G.; MARTINEZ, C. J.; REZENDE, C. Imunopatologia da Hanseníase: Aspectos Clínicos e Laboratoriais. Cidade: News Lab., p. 2, 2005.

SAVASSI, L. C. M. Hanseníase: políticas públicas e qualidade de vida de pacientes e seus cuidadores. Belo Horizonte: Cebtro de pesquisas Réne Rachou, p. $18,2010$.

SEPLAN. Secretaria de Estado de Planejamento e Coordenação Geral, Perfil do Município. Disponível em: http://www.seplan.mt.gov.br/ Acesso em: 14 de fev., 2014

SILVA, D. R. X.; IGNOTTI, E.; SANTOS, R. S.; HACON, S. S. Hanseníase, condições sociais e desmatamento na Amazônia brasileira. Revista Panamericana de Salud Pública, v. 21, p. 170 - 171, 2010.

SOUZA, C. S. Hanseníase: formas clínicas e diagnóstico diferencial. Medicina, Ribeirão Preto, v. 30, p. 325-334, 1997. 
ZAPPI, D.C.; SASAKI, D.; MILLIKEN, W.; IVA, J.; HENICKA, G.S.; BIGGS, N. \& FRISBY, S. Plantas vasculares da região do Parque Estadual Cristalino, Norte de Mato Grosso, Brasil. Acta Amazônica, v.41, p. 29-38, 2011. 